\title{
Bitcoin Poison? Anecdotal Evidence from Bitcoin Miners Revenue
}

\author{
David Spohn \\ Correspondence: David Spohn, Researcher, Lithia, Florida, USA. \\ Received: June 3, 2018 \\ doi:10.11114/aef.v5i4.3227 \\ Accepted: June 22, 2018 Available online: June 26, 2018 \\ URL: https://doi.org/10.11114/aef.v5i4.3227
}

\begin{abstract}
This paper explores the predictive qualities of Bitcoin Miners Revenue on Bitcoin Returns. Using data on Bitcoin in the cryptocurrency market from July 1, 2010 to February 20, 2018, we reflect intervariable correlations not previously examined. We analyze those relationships with a conditional regression analysis adjusting for calendar effects. We separate the sample, and use the last 17 trading days (month) to test a strategy based on the probability of Bitcoin Returns moving higher. After a slight modification to the logistic regression analysis, we find a profitable trading strategy exists based solely on Bitcoin Miners Revenue and the probability of Bitcoin Returns moving higher.
\end{abstract}

Keywords: cryptocurrency, returns, finance and investments

\section{Introduction}

The stardom of Bitcoin is prominent in circles ranging from illegal activity to speculative investors. While the literature on Bitcoin continues to emerge, the question of profitable investment strategies surfaces. We will not address the legitimacy of cryptocurrencies in this paper, but rather take the perspective of speculative investors to analyze the opportunity to use Bitcoin like other currency asset options. As exchanges continue to track Bitcoin and add the opportunity to trade them as investment tools (i.e. currency), it is more apparent that the body of literature needs to address such an opportunity.

At the core of Bitcoin (and cryptocurrencies in general) is the idea of eliminating the financial institution to process transactions, which adds an additional layer of cost to the party using the currency. Bitcoin and other cryptocurrencies attempt to mitigate this cost by using peer-to-peer transactions and open source software. Bitcoin miners solve complex mathematical problems to both unlock remaining Bitcoins, and verify Bitcoin transactions. Bitcoin miners are then rewarded financially (paid in Bitcoins) for completing the mathematical solutions before other miners in both revenue and fees. This unique structure (i.e. blockchain technology) creates an interesting valuation problem for Bitcoin and cryptocurrencies in general. Cryptography and a decentralized payment system seem to follow more closely with the decentralized foreign currency exchanges (excluding the banks) than traditional investments.

What determines Bitcoin market prices? Unlike stocks or foreign exchange, Bitcoin has no oversight other than the peer-to-peer network to monitor or oversee transactions (i.e. no regulatory entity). Do technical factors provide insight into the Bitcoin market? Do fundamental factors drive Bitcoin market price? Does investor sentiment (supply and demand) drive Bitcoin prices up and down? This literature focuses on short-term market inefficiencies and the lack of market fundamentals in Bitcoin pricing in the emerging cryptocurrency market.

Since Fama (1965) introduced the idea of the random walk hypothesis, academics have debated the idea of efficient versus inefficient markets. Clearly, the cryptocurrency market was not a function of Fama's hypothesis, and follows more closely with the idea of creative destruction (Schumpeter, 1942). While the debate of market efficiency or inefficiency continues, Spohn (2018) finds that short-term inefficiencies within the market can produce positive abnormal returns. Additionally, the foreign exchange literature is riddled with technical versus fundamental investment strategies with prevailing undertones of technical strategies leading to more profitable returns (Sweeney, 1986; Taylor \& Allen, 1992; Neeley, Weller, \& Ditmar, 1997; Marsh, 2000). Thus, we focus on short-term inefficiencies or non-fundamentals to predict Bitcoin returns.

The emerging literature on Bitcoin price prediction is relatively small. However, Shah and Zhang (2014) use a Bayesian regression to predict Bitcoin prices with success. Almeida, Tata, Moser, and Smit (2015) take a different approach by using an artificial neural network (ANN) to predict next day Bitcoin price using the previous day Bitcoin closing price and trading volume, while Indera, Yassin, Zabidi, and Rizman (2017) use a creative non-linear autoregressive with 
exogeneous input (NARX) to predict Bitcoin prices using opening, closing, minimum and maximum past Bitcoin prices with relative accuracy. Using short-term market inefficiencies, can we forecast Bitcoin returns? While recent studies have focused on more traditional variables and machine learning to predict Bitcoin returns, we focus on the impact of lagged Bitcoin Miners Revenue and its relationship to current Bitcoin returns. We fill a void in the literature by employing data on the Bitcoin market to gauge the effects of Bitcoin Miners Revenue on Bitcoin Returns and define a profitable trading strategy based on the probability of Bitcoin Returns moving higher.

\section{Theory and Hypotheses}

Classifying Bitcoin (or any cryptocurrency) as a traditional currency has inherent problems. The most prominent issue is determining the underlying value of a digital currency. Its unique structure is counter to traditional methodology, and therefore evaluating a 'virtual' currency by traditional means is challenging. Unlike traditional currency where value is tied to future expectations of country GDP (or in the past a gold standard), Bitcoin has no underlying value with which to base its current value. Expectations of valuation are increasingly predicated on the general populace and its willingness to use (transact or speculate) daily. While Bitcoin is slowly becoming more common in the marketplace, it is not a standard with which to transact.

Considering the lack of traditional fundamentals, Bitcoin aligns more theoretically with behavioral finance. Behavioral finance addresses the inefficiencies of the market by analyzing investor behavior. To accept behavioral finance conceptually as the theoretical lens, we must make certain assumptions. We assume that the cryptocurrency market is inefficient, and the Bitcoin speculators (i.e. investors) are irrational in their investment decisions. Schumpeter (1954) is one of the first to make note of the oddity of participants in the investment markets, while Shiller (1989) classifies this odd behavior as irrational. The irrational behavior exhibited by the speculators in the markets is defined as letting losing positions run, while closing profitable positions too quickly, thus extending losses, and reducing profit. While other works focus on the classification of trades (Lee \& Ready, 1991), order imbalance or volume (Menkhoff, Sarno, Schmeling, \& Schrimpf, 2016), we focus on Bitcoin miners and the relative difficulty to find a new block, thus unlocking new Bitcoin into the marketplace and its impact on the supply and demand for speculators in the Bitcoin market.

Given the relative newness of Bitcoin and the lack of underlying fundamentals, analyzing Bitcoin through the theoretical lens of behavioral finance, more specifically Bitcoin supply and demand seems to be the most logical approach. Focusing on the Bitcoin miners and their role in making new Bitcoins available for the general populace (up to 21 million Bitcoins) gives us insight into the cryptocurrency market not previously studied. Thus, we examine Bitcoin within the cryptocurrency market. While previous studies in similar markets examine price (Harris, 1987) and volume (Muller et. al, 1990), we focus on the Bitcoin miners' role in Bitcoin returns. We hypothesize the following:

Hypothesis 1a. As Bitcoin Difficulty increases, Bitcoin Miner Revenue increases, or a positive relationship between Bitcoin Difficulty and Bitcoin Miner Revenue.

Hypothesis 1b. As Bitcoin Difficulty increases, Bitcoin Transaction Fees increases, or a positive relationship between Bitcoin Difficulty and Bitcoin Transaction Fees.

We expect to see a positive relationship between Bitcoin Difficulty and both Miner Revenue and Transaction Fees to entice Bitcoin Miners to stay prevalent in their work. Motivation to keep mining Bitcoins for the general populace ensures greater circulation of Bitcoin in the marketplace, while rewarding Bitcoin Miners for their effort.

Hypothesis 2. As Bitcoin Difficulty increases, Bitcoin Market Price increases, or a positive relationship between Bitcoin Difficulty and Bitcoin Market Price.

We expect to see the Bitcoin Market Price increase relative to an increase in Bitcoin Difficulty. As miners spend more time and energy (effectively, cost) in mining, Bitcoin Market Price rises to compensate for the associated cost.

Hypothesis 3. As the lagged Change in Total Bitcoin increases, Bitcoin Trade Volume (reflected in total US Dollars) decreases, or a negative relationship between the lagged Change in Total Bitcoin and Bitcoin Trade Volume (reflected in US Dollars).

Under the conceptual lens of behavioral finance, we expect to see a decrease in trade volume (reflected in total US Dollars) as more Bitcoins enter the marketplace as miners solve the complex mathematical problems, whereby speculators perceive value diminishing, as the cryptocurrency (monetary) supply increases relative to demand (Cagan, 1958).

Hypothesis 4. A profitable trading strategy for Bitcoin speculation can be formulated, whereby entering a position and exiting a position can be clearly defined and profitable.

This literature examines Bitcoin variables through the theoretical lens of behavioral finance and focuses on short-term market inefficiencies and the lack of market fundamentals in Bitcoin pricing in the emerging cryptocurrency market. By 
examining the above relationships (Hypothesis 1a, 1b, 2, and 3), we propose a trading strategy (Hypothesis 4) for the Bitcoin marketplace by analyzing data from July 1, 2010 to February 20, 2018.

Due to the infancy of the Bitcoin market, this paper sheds light on previously unexplored relationships and enhances our understanding of the cryptocurrency market by examining non-fundamental effects.

\section{Data and Methodology}

\subsection{Data}

We collect daily Bitcoin data from two sources (https://coinmarketcap.com and https://blockchain.info). The collected daily Bitcoin data includes Bitcoin Market Price, Total Bitcoins, Bitcoin Trade Volume, Bitcoin Difficulty, Bitcoin Miners Revenue, and Bitcoin Transaction Fees from July 1, 2010 to February 20, 2018 (i.e. 2792 daily observations). Descriptive statistics are reflected in Table 1 (below) and include the sample mean, standard deviation, minimum and maximum values for the variables.

Table 1. Descriptive Statistics

\begin{tabular}{|c|c|c|c|c|}
\hline & Mean & Std & Min & $\operatorname{Max}$ \\
\hline \multicolumn{5}{|l|}{ Variables } \\
\hline Bitcoin Market Price & 943.48 & 2455.24 & .000 & 19498.68 \\
\hline Total Bitcoins & 11932152 & 3836511 & 3186200 & 16876825 \\
\hline Bitcoin Trade Volume & 86113761 & 318269622 & .000 & 5352015516 \\
\hline Bitcoin Difficulty & $1.9041 \mathrm{E}+11$ & $4.399 \mathrm{E}+11$ & 19.404 & $2.967 \mathrm{E}+12$ \\
\hline Bitcoin Miners Revenue & 2412716 & 5941326 & .000 & 53191582 \\
\hline Bitcoin Transaction Fees & 64.02 & 119.73 & .000 & 1495.95 \\
\hline
\end{tabular}

This table presents the descriptive statistics for the included variables from July 1, 2010 to February 20, 2018 (i.e. 2792 observations). Bitcoin Market Price is the calculated average in US Dollars across the major Bitcoin exchanges. Total Bitcoins is the total number of Bitcoins that have been mined. Bitcoin Trade Volume is the total value in US Dollars of trading volume on the major Bitcoin exchanges. Bitcoin Difficulty is the relative measure of the difficulty to find a new block to mine. Bitcoin Miners Revenue is defined as a monetary reward for unlocking Bitcoin blocks. Bitcoin Transaction Fees is the monetary reward for verifying Bitcoin transactions.

Table 1 reflects the sample mean of the daily Bitcoin Market Price across the major Bitcoin exchanges, effectively giving us the Bitcoin mid-price of 943.48 and a standard deviation of 2455.25 with a minimum value of .000 and a maximum value of 19498.68 (reflected in US Dollars). Given the unique nature of this study, capturing the beginning of the Bitcoin phenomenon, these values generally reflect daily expectations over the defined sample period.

The average Total Bitcoins (mined) is 11932152 and has a standard deviation of 3836 200. The minimum and maximum values are 3186200 and 16876825 respectively. The Bitcoin Trade Volume (reflecting the total value in US Dollars) mean is 86113761 with a standard deviation of 318269622 and minimum and maximum values of .000 and 5 352015516 (indicating an increased interest in both volume and monetary value). Bitcoin Difficulty is the reported relative measure of the difficulty of miners to find a new Bitcoin block with a mean of 190 billion, standard deviation of 439 billion, and minimum and maximum values of .000 and 2.967 trillion. The average Bitcoin Miners Revenue (payable in Bitcoins) is 2412716 with a standard deviation of 5941326 with minimum and maximum values of .000 and 53191 582, while the average Bitcoin Transaction Fees (payable in Bitcoins) is 64.02 with a standard deviation of 119.73 with minimum and maximum values of .000 and 1495.95 respectively. The data does not reflect abnormal data inconsistencies and generally falls in line with daily expectations over the examined period.

We use daily Bitcoin data following previous works (Froot \& Ramadorai, 2005; Spohn, 2018) as daily confidence bands have been shown to produce more consistency. Thus, we define the variables as such: Bitcoin Market Price is the calculated average in US Dollars across the major Bitcoin exchanges; Total Bitcoins is the total number of Bitcoins that have been mined (i.e. in circulation); Bitcoin Trade Volume is the total value in US Dollars of trading volume on the major Bitcoin exchanges; Bitcoin Difficulty is the relative measure of the difficulty to find a new block to mine; Bitcoin Miners Revenue is defined as the as a monetary reward for unlocking Bitcoin blocks; and Bitcoin Transaction Fees is the monetary reward for verifying Bitcoin transactions.

\subsection{Methodology}

We first explore the intervariable relationships by providing a correlation analysis between Bitcoin Returns, Bitcoin Market Price, the Change in Total Bitcoin, Bitcoin Trade Volume, Bitcoin Difficulty, Bitcoin Miners Revenue, and Bitcoin Transaction Fees. We define Bitcoin Returns as the lagged change in Bitcoin Market Price as the calculated change in Bitcoin Market Price from Day 1 to Day 2 and then subtracting 1. Thus, 


$$
\text { BitcoinReturns }_{t}=\left(\frac{\text { BitcoinMarketPrice }_{t}}{\text { BitcoinMarketPrice }_{t-1}}\right)-1
$$

where the Bitcoin Returns are calculated from day to day (day $t-1$ to day $t$ ). BitcoinReturns $s_{t}$ is the calculated exchange rate at the end of the day at time $t$, where the average Bitcoin exchange rate (effective mid-point) at time $t$ is BitcoinMarketPrice $_{t}$, and the average Bitcoin exchange rate (effective mid-point) at the end of the day at $t$ - 1 is BitcoinMarketPrice $_{t-1}$.

Figure 1 (below) reflects the daily Bitcoin Return for the period of July 1, 2010 to February 20, 2018.

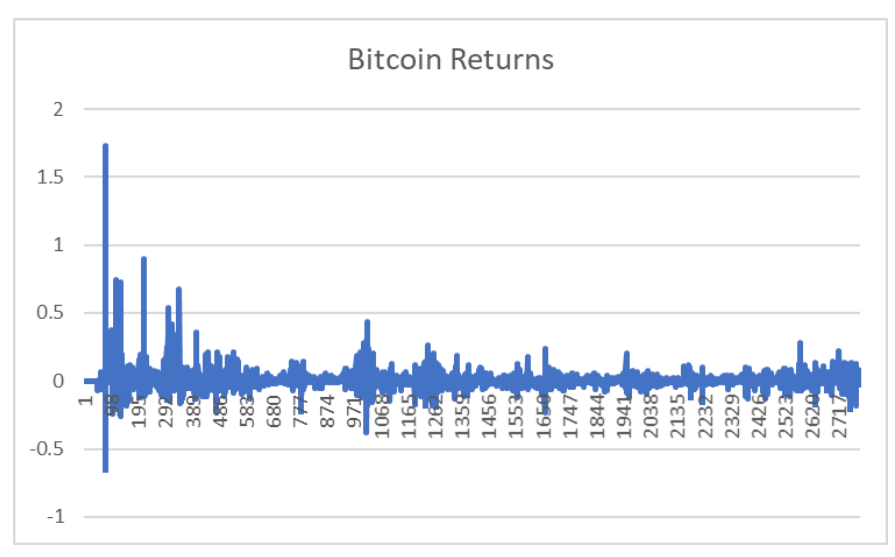

Figure 1. Bitcoin Returns

Bitcoin Market Price is defined as the calculated average in US Dollars across the major Bitcoin exchanges (effectively the mid-price), while the Change in Total Bitcoin is the calculated change in Total Bitcoins (actual number of Bitcoins mined). We calculate the change from Day 1 to Day 2 and subtract 1 from the difference. Thus, our equation is:

$$
\Delta{\text { in } \text { TotalBitcoin }_{t}}=\left(\frac{\text { TotalBitcoin }_{t}}{\text { TotalBitcoin }_{t-1}}\right)-1
$$

where the change in Total Bitcoin are calculated from day $t-1$ to day $t$. $\Delta$ in TotalBitcoin Th $_{t}$ is calculated change in Total Bitcoin (in circulation) at the daily close at time $t$. TotalBitcoin $t$ is the Total Bitcoin (in circulation) at time $t$, and the Total Bitcoin (in circulation) at the daily close at $t-1$ is TotalBitcoin $_{t-1}$.

We define Bitcoin Trade Volume as the total value in US Dollars of trading volume on the major Bitcoin exchanges. Bitcoin Difficulty is the relative measure of the difficulty to find a new block to mine, while Bitcoin Miners Revenue is the as a monetary reward for unlocking Bitcoin blocks, and Bitcoin Transaction Fees is the monetary reward for verifying Bitcoin transactions.

We explore these relationships with a correlation analysis and report the Pearson's correlation coefficient (Hypothesis 1a, 1b, 2, and 3). Intervariable relationship dependent, we run a regression analysis to determine the ability to predict current Bitcoin Returns. Thus, we define the regression as:

$$
\text { BitcoinReturns }_{t}=\alpha_{i}+\beta_{1} X_{1}+\beta_{2} X_{2}+\beta_{3} X_{3}+\beta_{4} X_{4}+\varepsilon_{i}
$$

where BitcoinReturns $s_{t}$ is the dependent variable of interest and defined as the Bitcoin Returns at time $t$. We use the remaining variables as predictors and define $X_{1}$ as Bitcoin Trade Volume, $X_{2}$ as Bitcoin Difficulty, $X_{3}$ as the Bitcoin Miners Revenue, and $X_{4}$ as Bitcoin Transaction Fees. $\beta$ s are the reported regression coefficients, while $\alpha_{i}$ is the constant (intercept) and $\varepsilon_{i}$ is the error term.

We also run regressions on intervals consistent with capturing calendar effects reported in financial markets. To capture these effects, we run regressions on current Bitcoin Returns against the variables for the calendar timeframes: Daily, Mondays, Tuesdays, Wednesdays, Thursdays, Fridays, and Weekends.

Pending the outcome of the significance levels of the reported variables in the previous analyses, we run a complex logistic regression to create a trading rule, where we determine the likelihood of Bitcoin Returns moving higher. We define the logit model as:

$$
\operatorname{logit}\left(\text { higher returns) }=\ln \left[\frac{\pi}{1-\pi}\right]=\alpha+\beta_{1} X_{1}+\beta_{2} X_{2}+\beta_{3} X_{3}+\beta_{4} X_{4}\right.
$$

therefore,

$$
\pi=\text { Probability (higher returns } \mid X_{1}=x_{1}, X_{2}=x_{2}, X_{3}=x_{3}, X_{4}=x_{4} \text { ) }
$$




$$
=\frac{\mathrm{e}^{\alpha+\beta_{1} X_{1}+\beta_{2} X_{2}+\beta_{3} X_{3}+\beta_{4} X_{4}}}{1+\mathrm{e}^{\alpha+\beta_{1} X_{1}+\beta_{2} X_{2}+\beta_{3} X_{3}+\beta_{4} X_{4}}}
$$

where $\pi$ is the probability of Bitcoin Returns moving higher. $X_{1}$ is Bitcoin Trade Volume; $X_{2}$ is Bitcoin Difficulty; $X_{3}$ is Bitcoin Miners Revenue; and $X_{4}$ is Bitcoin Transaction Fees. $\alpha$ is the constant (Y intercept), while the $\beta$ s are the regression coefficients. Consistent with previous literature, we use the maximum likelihood method to estimate both the $\alpha$ and the $\beta$ s (Schlesselman, 1982; Haberman, 1978).

We first run a correlation analysis to explore the intervariable relationships (Hypotheses 1a, 1b, 2, and 3). Then, we regress the variables of interest against Bitcoin Returns with varying calendar effects to determine the ability to predict Bitcoin Returns. We use this information to create a trading rule using a complex logistic regression to determine the likelihood of Bitcoin Returns moving higher (Hypothesis 4). We use the accumulated data to forecast the probability of Bitcoin Returns moving higher for the last 17 trading days (of the dataset) and analyze the results.

\section{Empirical Results}

\subsection{Correlation Results}

We examine the correlation analysis to determine the intervariable relationships of Bitcoin Returns, Bitcoin Market Price, the Change in Total Bitcoin, Bitcoin Trade Volume, Bitcoin Difficulty, Bitcoin Miners Revenue, and Bitcoin Transaction Fees and report the Pearson's correlation coefficient.

Table 2. Correlation Analysis for the Variables of Interest

\begin{tabular}{l|c|c|c|c|c|c}
\hline Variable & 1 & 2 & 3 & 4 & 5 & 6 \\
\hline 1. Bitcoin Returns & & & & & & \\
\hline 2. Bitcoin Market Price & .006 & & & & & \\
\hline 3. Change in Total Bitcoin & $.077^{* *}$ & $-.278^{* *}$ & & & & \\
\hline 4. Bitcoin Trade Volume & .000 & $.866^{* *}$ & $-.200^{* *}$ & & & \\
\hline 5. Bitcoin Difficulty & -.004 & $.900^{* *}$ & $-.328^{* *}$ & $.764^{* *}$ & & \\
\hline 6. Bitcoin Miners Revenue & .000 & $.985^{* *}$ & $-.276^{* *}$ & $.868^{* *}$ & $.836^{* *}$ & \\
\hline 7. Bitcoin Transaction Fees & -.009 & $.777^{* *}$ & $-.338^{* *}$ & $.698^{* *}$ & $.717^{* *}$ & $.780^{* *}$ \\
\hline
\end{tabular}

** Correlation is significant at the 0.01 level (2-tailed). * Correlation is significant at the 0.05 level (2-tailed).

Table 2 reports the Pearson's correlation coefficient and represents the intercorrelations of the variables used in this study. Bitcoin Returns is defined as the calculated change in Bitcoin Market Price by subtracting 1 from the difference between Day 1 to Day 2. Bitcoin Market Price is the calculated average in US Dollars across the major Bitcoin exchanges. Change in Total Bitcoin is the calculated change in Total Bitcoins (total number of Bitcoins mined) from Day 1 to Day 2 and then subtracting 1 from the difference. Bitcoin Trade Volume is the total value in US Dollars of trading volume on the major Bitcoin exchanges. Bitcoin Difficulty is the relative measure of the difficulty to find a new block to mine. Bitcoin Miners Revenue is defined as a monetary reward for unlocking Bitcoin blocks. Bitcoin Transaction Fees is the monetary reward for verifying Bitcoin transactions.

The correlation analysis yields some interesting results. Supporting Hypothesis $1 \mathrm{a}$ and $1 \mathrm{~b}$, we find that as Bitcoin Difficulty increases, both Bitcoin Miner Revenue and Bitcoin Transaction Fees increase (both significant at the $\mathrm{p}<.01$ levels). The reported Pearson's correlation coefficients are $r=.836$ and $r=.717$, respectively. We also find support for Hypothesis 2, where Bitcoin Difficulty increases, Bitcoin Market Price also increases, or a positive relationship. The reported Pearson's correlation coefficient is $r=.900$, significant at the $p<.01$ level. Additionally, we find support for Hypothesis 3, where the Change in Total Bitcoin increases, Bitcoin Trade Volume decreases. The Pearson's correlation coefficient is $r=-.200$, significant at the $p<.01$ level.

Bitcoin Returns are relatively insignificant to the other variables, except for the Change in Total Bitcoin $(r=.077$ and significant at the $\mathrm{p}<.01$ level). The relationships between Bitcoin Returns and Bitcoin Difficulty $(\mathrm{r}=-.004)$ as well as Bitcoin Transaction Fees $(r=-.009)$ are negative and insignificant. Bitcoin Market Price is negatively correlated to the Change in Total Bitcoin $(r=-.278$, significant at $\mathrm{p}<.01)$, while positively correlated to Bitcoin Trade Volume $(\mathrm{r}=.866$, significant at $\mathrm{p}<.01)$, Bitcoin Miners Revenue $(\mathrm{r}=.985$, significant at $\mathrm{p}<.01)$, and Bitcoin Transaction Fees $(\mathrm{r}=.777$, significant at $\mathrm{p}<.01)$. The Change in Total Bitcoin is negatively correlated to Bitcoin Difficulty $(r=-.328$, significant at $\mathrm{p}<.01)$, Bitcoin Miners Revenue $(\mathrm{r}=-.276$, significant at $\mathrm{p}<.01)$, and Bitcoin Transaction Fees $(\mathrm{r}=-.338$, significant at $\mathrm{p}<.01)$.

\subsection{Regression Results}

We regress Bitcoin Returns against the predictor variables of Bitcoin Trade Volume, Bitcoin Difficulty, Bitcoin Miners Revenue, and Bitcoin Transaction Fees to determine how well the data performs. To measure calendar effects on the regression, we first analyze the dataset on the holistic data (daily), before running the regression values for: Mondays, 
Tuesdays, Wednesdays, Thursdays, Fridays, and Weekends (Friday close through Sunday open).

Table 3. Regression Analysis for Bitcoin Returns

\begin{tabular}{|c|c|c|c|c|c|c|c|}
\hline Period & D & Mon & Tue & Wed & Thu & Fri & Wk \\
\hline Constant (Intercept) & $.009 * * *$ & $.007^{*}$ & $.011^{* *}$ & .011 & .006 & $.009 * *$ & $.007^{* *}$ \\
\hline Bitcoin Trade Volume (a) & $5.789 \mathrm{E}-14$ & $2.681 \mathrm{E}-12$ & $5.566 \mathrm{E}-13$ & $-4.253 \mathrm{E}-12$ & $1.418 \mathrm{E}-11$ & $-8.669 \mathrm{E}-12$ & $-4.415 \mathrm{E}-11$ \\
\hline Bitcoin Difficulty (b) & $-2.990 \mathrm{E}-14^{* *}$ & $-3.241 \mathrm{E}-14^{*}$ & $-2.410 \mathrm{E}-14$ & $-1.436 \mathrm{E}-14$ & $-5.212 \mathrm{E}-14^{*}$ & $-3.159 \mathrm{E}-14$ & $-2.454 \mathrm{E}-14$ \\
\hline Bitcoin Miners Revenue (c) & $-6.174 \mathrm{E}-9 * * *$ & $-5.036 \mathrm{E}-9$ & $-3.328 \mathrm{E}-9$ & $-4.600 \mathrm{E}-9$ & $-1.314 \mathrm{E}-8^{* *}$ & $-2.404 \mathrm{E}-9$ & $-5.090 \mathrm{E}-9$ \\
\hline Bitcoin Transaction Fees (d) & $-3.005 \mathrm{E}-6$ & $6.645 \mathrm{E}-5$ & $4.206 \mathrm{E}-5$ & $-4.514 \mathrm{E}-5$ & $5.354 \mathrm{E}-5$ & $-6.421 \mathrm{E}-5$ & $-3.458 \mathrm{E}-5$ \\
\hline \multirow[t]{5}{*}{ Model } & $\mathrm{Y}=5.789 \mathrm{E}-14 \mathrm{a}$ & $\mathrm{Y}=.2 .681 \mathrm{E}-12 \mathrm{a}+-$ & $\mathrm{Y}=5.566 \mathrm{E}-13$ & $\mathrm{Y}=-4.253 \mathrm{E}-12 \mathrm{a}+-1$ & $\mathrm{Y}=1.418 \mathrm{E}-11$ & $Y=8.669 E-12 a+-$ & $\mathrm{Y}=-4.415 \mathrm{E}-11 \mathrm{a}+-$ \\
\hline & $+-2.990 \mathrm{E}-14 \mathrm{~b}+$ & $3.241 \mathrm{E}-14 \mathrm{~b}+-5.0$ & $\mathrm{a}+-2.410 \mathrm{E}-14$ & $.436 \mathrm{E}-14 \mathrm{~b}+-4.600$ & $\mathrm{ab}+-5.212 \mathrm{E}-1$ & $3.159 \mathrm{E}-14 \mathrm{~b}+-2.4$ & $2.454 \mathrm{E}-14 \mathrm{~b}+-5.0$ \\
\hline & $-6.174 \mathrm{E}-9 \mathrm{c}+-3$ & $36 \mathrm{E}-9 \mathrm{c}+6.645 \mathrm{E}-5$ & $\mathrm{~b}+-3.328 \mathrm{E}-9 \mathrm{c}$ & E-9c+-4.514E-5d+ & $4 \mathrm{~b}+-1.314 \mathrm{E}-8$ & $04 \mathrm{E}-9 \mathrm{c}+-6.421 \mathrm{E}-$ & $90 \mathrm{E}-9 \mathrm{c}+-3.458 \mathrm{E}-$ \\
\hline & $005 \mathrm{E}-6 \mathrm{~d}+.009$ & $\mathrm{~d}+.007$ & $+4.206 \mathrm{E}-5 \mathrm{~d}$ & .011 & $c+5.354 \mathrm{E}-5 \mathrm{~d}$ & $5 \mathrm{~d}+.009$ & $5 \mathrm{~d}+.007$ \\
\hline & & & +.011 & & +.006 & & \\
\hline PredictedY & .009 & .0071 & .0111 & .011 & .0061 & .009 & .007 \\
\hline
\end{tabular}

Significance levels are reported as: *,**,***.05, .01 and .001 levels, respectively.

Table 3 reflects the values of the regression for the Constant (Intercept), Bitcoin Trade Volume (Predictor), Bitcoin Difficulty (Predictor), Bitcoin Miners Revenue (Predictor), Bitcoin Transaction Fees (Predictor), Model (Regression), and the Predicted Value of Y for the dataset inclusive of the dates July 1, 2010 through February 20, 2018. To capture calendar effects, we separate the data into periods where we consider the overall Daily regression (designated as ' $\mathrm{D}$ '), and measure the effects for each day of the week: Mondays (Mon), Tuesdays (Tue), Wednesdays (Wed), Thursdays (Thu), Fridays (Fri), and Weekends (Wk). Weekends are measured from Friday's close to Sundays open (effectively gap opens/closes from Friday to Sunday).

The regressions in the appointed timeframes are all positive. Within the Daily column, we find the Constant (Intercept) is positive and significant at the $\mathrm{p}<.001$ level. Bitcoin Trade Volume is positive, but insignificant at acceptable levels. Bitcoin Difficulty is negative and significant at the $\mathrm{p}<.01$ level, while Bitcoin Miners Revenue is negative and significant at the $\mathrm{p}<.001$ level. Bitcoin Transaction Fees is negative and insignificant. The calendar effects seem to lack significant predictors, as the Constant (Intercept) is positive in all periods and significant on Monday $(\mathrm{p}<.05)$, Tuesday $(\mathrm{p}<.01)$, Friday $(\mathrm{p}<.01)$, and Weekends $(\mathrm{p}<.01)$. Bitcoin Difficulty is negative across all periods but only significant on Monday ( $\mathrm{p}<.05)$, and Thursday $(\mathrm{p}<.05)$. Bitcoin Trade Volume is insignificant and varies across the periods, while only 4 periods are positive (Daily, Mondays, Tuesdays and Thursdays). Bitcoin Miners Revenue is negative across all periods, and only significant in the Daily and Thursday periods. Bitcoin Transaction Fees is insignificant across all periods, while only 4 periods are negative (Daily, Wednesdays, Fridays, and Weekends). The discrepancy in significant relationships across the calendar effects compared to the Daily regressions imply potential momentum effects when isolated. While reflecting significant relationships in the Daily regressions, the regressions overall do a relatively poor job in predicting Bitcoin Returns. Therefore, we focus on the logistic regression model in determining the probability of Bitcoin Returns moving higher.

\subsection{Logistic Regression Results}

Given the regression results, we focus on the significant relationships expressed in the Daily regressions. Thus, we focus on Bitcoin Difficulty and Bitcoin Miners Revenue to develop a trading rule. We adjust the logistic regression from equations (4), (5), and (6) to reflect the following:

$$
\operatorname{logit}(\text { higher returns })=\ln \left[\frac{\pi}{1-\pi}\right]=\alpha+\beta_{1} X_{1}+\beta_{2} X_{2}
$$

therefore,

$$
\pi=\text { Probability (higher returns } \mid X_{1}=x_{1}, X_{2}=x_{2} \text { ) }
$$




$$
=\frac{\mathrm{e}^{\alpha+\beta_{1} X_{1}+\beta_{2} X_{2}}}{1+\mathrm{e}^{\alpha+\beta_{1} X_{1}+\beta_{2} X_{2}}}
$$

where $\pi$ is the probability of Bitcoin Returns moving higher. $X_{1}$ is Bitcoin Difficulty and $X_{2}$ is Bitcoin Miners Revenue. $\alpha$ is the constant (Y intercept), while the $\beta$ s are the regression coefficients. Consistent with previous literature, we use the maximum likelihood method to estimate both the $\alpha$ and the $\beta$ s (Schlesselman, 1982; Haberman, 1978).

We take the natural log of both Bitcoin Difficulty and Bitcoin Miners Revenue to scale the data (Fama, 1984). We measure the change in both Bitcoin Difficulty and Bitcoin Miners Revenue from day 1 to day 2 by calculating each variable value: (natural $\log$ of Bitcoin Difficulty Day 2/natural $\log$ of Bitcoin Difficulty Day 1) - 1 and (natural $\log$ of Bitcoin Miners Revenue Day 2/natural log of Bitcoin Miners Revenue Day 1) - 1 .

We evaluate 4 models in the logistic regression, where model one includes the variable of the natural log of the change in Bitcoin Difficulty; model two includes the variable of the natural log of the change in Bitcoin Miners Revenue; model three includes the variables of the natural log of the change in Bitcoin Miners Revenue and the natural log of the change in Bitcoin Difficulty; and model four includes the natural log of the change in Bitcoin Miners Revenue, the natural log of the change in Bitcoin Difficulty, and the interaction of the natural log of the change in Bitcoin Miners Revenue and the natural $\log$ of the change in Bitcoin Difficulty.

Based on the Omnibus Test of Model Coefficients, we choose the first model where the natural log of the change in Bitcoin Miners Revenue is the only independent variable of the likelihood of Bitcoin Returns moving higher. The other three models do not add any significance to model two: $\chi^{2}(1)=149.192, p=.001$.

Table 4. Logistic Regression Analysis

\begin{tabular}{lcccccc}
\hline Predictor & $\beta$ & SE $\beta$ & Wald's $\chi^{2}$ & $d f$ & $p$ & $\mathrm{e}^{\beta}$ (odds ratio) \\
\hline Constant & .116 & .039 & 8.651 & 1 & .003 & $1.113 \mathrm{E}-18$ \\
$\Delta$ in Bitcoin Miners Revenue & 41.554 & 3.69 & 126.835 & 1 & .001 & 1.122 \\
\hline Test & & & $\chi^{2}$ & $d f$ & $p$ & \\
\hline $\begin{array}{l}\text { Overall Model Evaluation } \\
\quad \text { Likelihood Ratio Test }\end{array}$ & & 149.92 & 1 & .001 & \\
$\begin{array}{l}\text { Goodness-of-fit Test } \\
\quad \text { Hosmer \& Lemeshow }\end{array}$ & & & & & \\
\hline
\end{tabular}

Table 4 reports the logistic regression including the predictor variable of the natural log of the change in Bitcoin Miners Revenue from July 1, 2010 to 25 January 2018. Table 4 reflects the coefficients, chi-squares, degrees of freedom, significance, and the odds ratio, as well as the overall model evaluation and goodness-of-fit.

We find that the model's overall evaluation (likelihood ratio test) fits the data well (significant at the $\mathrm{p}<.001$ level). The goodness-of-fit test (Hosmer and Lemeshow) implies a reasonably good model fit, as the insignificant result $(\mathrm{p}>.05)$ yields an obtainable null hypothesis. The natural $\log$ of the change in Bitcoin Miners Revenue is significant $(\mathrm{p}$ $<.001)$ as is the Constant $(\mathrm{p}<.01)$.

Table 5. Observed and Predicted Frequencies of Bitcoin Returns moving higher

\begin{tabular}{lccc}
\hline & Predicted & & \\
Observed & Yes & Predicted No & Correct \\
\hline Yes & 1035 & 423 & $71.0 \%$ \\
No & 671 & 615 & $47.8 \%$ \\
Overall \% Correct & & & $60.1 \%$ \\
\hline
\end{tabular}

Sensitivity $=1035 /(10353+423)=71.0 \%$, Specificity $=615 /(615+671)=47.8 \%$, False Positives $=671 /(671+1035)=39.3 \%$, False Negatives $=423 /(423+615)=40.8 \%$

Table 5 summarizes the observed and predicted frequencies of Bitcoin Returns moving higher. Based on the natural log of the change in Bitcoin Miners Revenue (predictor), we see that the model does slightly better than predicting the Bitcoin Returns moving higher $50 \%$ of the time $(60.1 \%$ overall percentage correct). The model reflects false positives of $39.3 \%$, while false negatives are slightly worse at $40.8 \%$.

\subsection{Trading Strategy Test Results}

The logistic regression based on the natural log of the change in Bitcoin Miners Revenue does slightly better than guessing $(60.1 \%$ overall percentage correct). Based solely on the logistic regression, the profitability depends on gains outpacing losses to remain profitable (holistically). However, given slight modifications, the trading strategy can be 
improved.

We test our strategy by taking the logistic regression model above, and make a minor modification where if the probability of Bitcoin Returns is forecasted higher than the previous day, we Buy. Conversely, if the probability of Bitcoin Returns moving higher is lower than the previous day, we Sell.

Table 6. Predicted Probability of Bitcoin Returns (January 26, 2018 to February 19, 2018)

\begin{tabular}{lcccc}
\hline & $\begin{array}{c}\Delta \text { in Bitcoin Miners } \\
\text { Revenue } \\
\beta=41.554\end{array}$ & $\begin{array}{c}\text { Intercept } \\
=.116\end{array}$ & $\begin{array}{c}\text { Predicted } \\
\text { Probability of } \\
\text { Higher Returns }\end{array}$ & $\begin{array}{c}\text { Actual Outcome of } \\
\text { Higher Returns } \\
1=\text { Yes, } 0=\text { No }\end{array}$ \\
Date & -.003 & .116 & 1.9853 & 0 \\
\hline January 26, 2018 & -.006 & .116 & 1.7210 & 0 \\
January 29, 2018 & .001 & .116 & 2.3687 & 0 \\
January 30, 2018 & -.019 & .116 & .9883 & 0 \\
January 31, 2018 & .012 & .116 & 3.6990 & 0 \\
February 1, 2018 & .003 & .116 & 2.5513 & 0 \\
February 2, 2018 & -.009 & .116 & 1.5606 & 1 \\
February 5, 2018 & .002 & .116 & 2.4359 & 1 \\
February 6, 2018 & -.008 & .116 & 1.5790 & 1 \\
February 7, 2018 & -.004 & .116 & 1.8894 & 1 \\
February 8, 2018 & .004 & .116 & 2.6368 & 0 \\
February 9, 2018 & .009 & .116 & 3.2731 & 1 \\
February 12, 2018 & -.002 & .116 & 2.0484 & 1 \\
February 13, 2018 & .010 & .116 & 3.4042 & 1 \\
February 14, 2018 & -.014 & .116 & 1.2516 & 1 \\
February 15, 2018 & .011 & .116 & 3.5845 & 1 \\
February 16, 2018 & .004 & .116 & 2.5985 & 1 \\
February 19, 2018 & & &
\end{tabular}

Table 6 summarizes the predicted probability of Bitcoin Returns for the above dates. Based on the predicted probability of Bitcoin Returns moving higher, the Bitcoin market is in an uptrend. A 'buy and hold' strategy seems appropriate for this type of market. However, utilizing the new trading rule based on the logistic regression, profits increase by $843 \%$ during the same period. Entering and exiting the market based on an increase (decrease) in probability of Bitcoin Returns moving higher or lower yields $\$ 3551.58$ (US Dollars) per Bitcoin compared to $\$ 420.58$ per Bitcoin (US Dollars) with the 'buy and hold' strategy for the period of January 26, 2018 to February 19, 2018, thus supporting Hypothesis $4 .{ }^{1}$

\section{Robustness Tests}

We look to see if there is an additional variable related to Bitcoin Returns. Given the unique nature of this study, the only feasible variable to compare is interest rates. The daily frequency of the Bitcoin dataset works well with the daily frequency of interest rate yields. We take the one-month constant maturity yields of the US Treasury Securities (www.federalreserve.gov). We compare this data by running a correlation analysis between the lagged change in Bitcoin Returns (Eq. (1)) and the difference in interest rate changes daily. Thus, we define the change in simple interest rates as:

$$
\text { Interest }_{\text {Rate }}=\text { interestrate }_{t}-\text { interestrate }_{t-1}
$$

where we compute the difference in the interest rate from day $t-1$ to day $t$. InterestRate $e_{t}$ is the product of the change in the daily close, where interestrate ${ }_{t}$ is the interest rate close at time $t$, while the interest rate close at day $t-1$ is interestrate $_{t-1}$.

We run a correlation analysis between the change in the simple interest rate and the lagged change in Bitcoin Returns for the same time periods as Table 3. While none of the correlations are significant, we do find that Daily $(\mathrm{r}=.001)$, Tuesdays $(\mathrm{r}=.003)$, and Wednesdays $(\mathrm{r}=.019)$ reflect positive correlations. Mondays $(\mathrm{r}=-.005)$, Thursdays $(\mathrm{r}=-.040)$, Fridays $(\mathrm{r}=-.072)$, and Weekends $(\mathrm{r}=-.044)$ all reflect negative correlations. This lends more credibility to the absence of fundamentals driving the cryptocurrency market.

Even though the relationship between Bitcoin Miners Revenue and Bitcoin Transaction Fees is relatively strong ( $\mathrm{r}$ $=.780)$ and significant $(\mathrm{p}<.01)$, the discrepancy in monetary value is large (based on the descriptive statistics). As the Bitcoin market rapidly approaches the benchmark of 21 million (total Bitcoin), Bitcoin Transaction Fees will be the source of revenue for miners in the market.

\footnotetext{
${ }^{1}$ Bitcoin exhibited traits of a strong uptrend based on the logistic regression over the extended period. During that period, the 'buy and hold' strategy is more profitable versus the proposed modified logistic regression strategy when compared to the last year of the analysis (February 2017 to February 2018).
} 


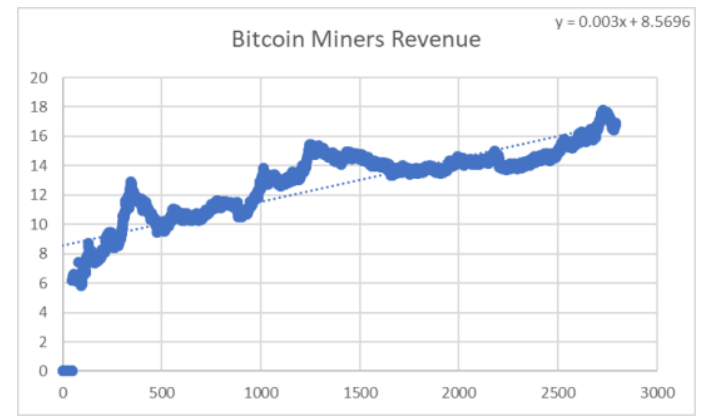

Figure 2. Bitcoin Miners Revenue

We take the natural log of the Bitcoin Miners Revenue and compare it to the natural log of the Bitcoin Transaction Fees, as eventually the miner's revenue will diminish for introducing more Bitcoins into the market.

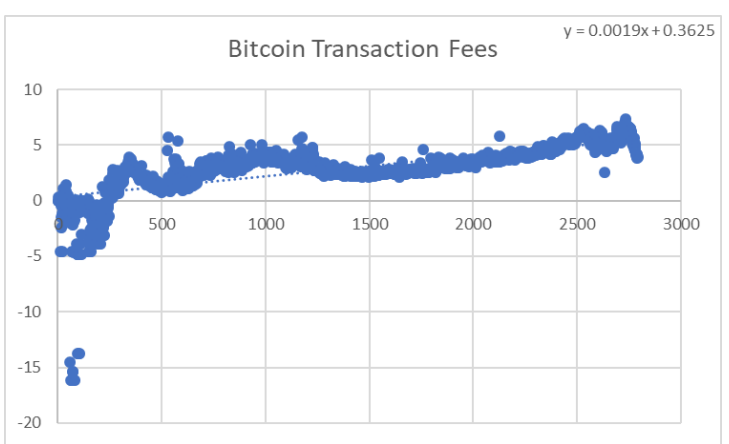

Figure 3. Bitcoin Transaction Fees

We observe that through the period analyzed (July 1, 2010 through February 20, 2018), Bitcoin Miner Revenue is growing at a faster rate than Bitcoin Transaction Fees. This is counter-intuitive to the idea that Bitcoin will replace more traditional means of currency interactions, as well as lend support to the idea that supply and demand are (at least in part) an underlying factor behind the rapid rise in miner revenue.

\section{Conclusion}

Our analysis reflects the valuable information Bitcoin Miners Revenue contains when compared to Bitcoin Returns in the emerging cryptocurrency market. Using a novel dataset (https://coinmarketcap.com and https://blockchain.info) from July 1, 2010 to February 20, 2018, we show unique intervariable relationships not previously explored. We use the theoretical lens of behavioral finance and build on the intervariable relationships while adjusting for calendar effects to develop a trading rule based on the probability of Bitcoin Returns moving higher (logistic regression). We show that the Bitcoin data reflects unique relationships, and that a profitable trading strategy can be formulated around those relationships. Our findings highlight previous literature on trading strategies, while differentiating by focusing on Bitcoin Miners Revenue to predict Bitcoin Returns.

We find support for all the hypotheses but do urge caution in this emerging market. Warren Buffet was recently quoted as saying about Bitcoin: 'it's probably rat poison squared. ${ }^{2,}$ We find undertones of support for the lack of fundamentals within this market (in line with behavioral finance), but the cryptocurrency market is unique. Bitcoin particularly has a limit on the number of Bitcoins in circulation (maximum of 21 million). As of this paper, we are rapidly approaching that number (approximately 17 million). Once the 21 million Bitcoins are released, we suspect that supply and demand will ultimately determine the value of the Bitcoin market. If the general populace fails to accept Bitcoin as a common currency with which to transact, we suspect that the value will dramatically diminish.

Limitations of this study include an emerging market with data capturing the beginning or introduction of Bitcoin. The data capturing the introduction of Bitcoin into the marketplace may ultimately prove inaccurate (outlier) or unduly influencing outcomes. Future studies will benefit from a more robust dataset. More insightful outcomes and analyses will transpire as additional research is added to the literature.

\footnotetext{
${ }^{2}$ In an interview with CNBC's Becky Quick, Berkshire Hathaway's chairman and CEO, Warren Buffet warned potential investors about cryptocurrencies on May 5, 2018

(https://www.cnbc.com/2018/05/05/warren-buffett-says-bitcoin-is-probably-rat-poison-squared.html).
} 


\section{References}

Almeida, J., Tata, S., Moser, A., \& Smit, V. (2015). Bitcoin Prediction using ANN.

Cagan, P. (1958). The Demand for Currency Relative to the Total Money Supply. Journal of Political Economy, 66(4), 303-328. https://doi.org/10.1086/258056

Fama, E. F. (1965). The Behavior of Stock Market Prices. Journal of Political Economy, 38(1), 34-105. https://doi.org/10.1086/294743

Fama, E. F. (1984). Forward and Spot Exchange Rates. Journal of Monetary Economics, 14(3), $319-338$. https://doi.org/10.1016/0304-3932(84)90046-1

Froot, K. A., \& Ramadorai, T. (2005). Currency Returns, Intrinsic Value, and Institutional-Investor Flows. Journal of Finance, 60(3), 1535-1566. https://doi.org/10.1111/j.1540-6261.2005.00769.x

Haberman, S. J. (1978). Analysis of qualitative data, 1.

Harris, L. (1987). Transaction Data Tests of the Mixture of Distributions Hypothesis. Journal of Financial and Quantitative Analysis, 22(2), 127-141. https://doi.org/10.2307/2330708

Indera, N. I., Yassin, I. M., Zabidi, A., \& Rizman, Z. I. (2017). Non-linear Autoregressive with Exogenous Input (NARX) Bitcoin Price Prediction Model using PSO-optimized Parameters and Moving Average Technical Indicators. Journal of Fundamental and Applied Sciences, 9(3S), 791-808. https://doi.org/10.4314/jfas.v9i3s.61

Lee, C., \& Ready, M. J. (1991). Inferring Trade Directions from Intraday Data. Journal of Finance, 46(2), $733-746$. https://doi.org/10.1111/j.1540-6261.1991.tb02683.x

Marsh, I. W. (2000). High-Frequency Markov Switching Models in the Foreign Exchange Market. Journal of $\begin{array}{lll}\text { Forecasting, } & 19(2), & 123-134 .\end{array}$ https://doi.org/10.1002/(SICI)1099-131X(200003)19:2<123::AID-FOR750>3.0.CO;2-C

Menkhoff, L., Sarno, L., Schmeling, M., \& Schrimpf, A. (2016). Information flows in Foreign Exchange Markets: Dissecting Customer Currency Trades. Journal of Finance, 71(2), 601-634. https://doi.org/10.1111/jofi.12378

Muller, U. A., Dacorogna, M. M., Olsen, R. B., Pictet, O. V., Schwarz, M., \& Morgenegg, C. (1990). Statistical Study of Foreign Exchange Rates, Empirical Evidence of a Price Change Scaling Law and Intraday Analysis. Journal of Banking of Finance, 14(6), 1189-1208. https://doi.org/10.1016/0378-4266(90)90009-Q

Neely, C., Weller, P., \& Dittmar, R. (1997). Is Technical Analysis in the Foreign Exchange Market Profitable? A Genetic Programming Approach. The Journal of Financial and Quantitative Analysis, 32(4), 405-426. https://doi.org/10.2307/2331231

Schlesselman, J. J. (1982). Case control studies: Design, control, analysis. New York: Oxford University Press.

Schumpeter, J. A. (1942). Socialism, Capitalism and Democracy. Harper and Brothers.

Schumpeter, J. A. (1954). History of Economic Analysis. New York: Oxford University Press.

Shah, D., \& Zhang, K. (2014, September). Bayesian Regression and Bitcoin. In Communication, Control, and Computing (Allerton), $201452^{\text {nd }}$ Annual Allerton Conference on (pp. 409-414). IEEE. https://doi.org/10.1109/ALLERTON.2014.7028484

Shiller, R. J. (1989). Investor Behavior in the October 1987 Stock Market Crash: Survey Evidence.

Spohn, D. (2018). Does Foreign Exchange Volume Activity Predict Foreign Exchange Returns? Evidence from Buy-Sell Volume in the Euro-Dollar Market. Forthcoming, Applied Finance and Accounting, 4(2), 15-28. https://doi.org/10.11114/afa.v4i2.3169

Sweeney, R. J. (1986). Beating the Foreign Exchange Market. The Journal of Finance, 41(1), 163-182. https://doi.org/10.1111/j.1540-6261.1986.tb04497.x

Taylor, M. P., \& Allen, H. (1992). The Use of Technical Analysis in the Foreign Exchange Market. Journal of International Money and Finance, 11(3), 304-314. https://doi.org/10.1016/0261-5606(92)90048-3

\section{Copyrights}

Copyright for this article is retained by the author(s), with first publication rights granted to the journal.

This is an open-access article distributed under the terms and conditions of the Creative Commons Attribution license which permits unrestricted use, distribution, and reproduction in any medium, provided the original work is properly cited. 